\title{
Photonic band mixing in linear chains of optically coupled micro-spheres
}

\author{
L. I. Deych and A. Roslyak \\ Physics Department, Queens College, \\ City University of New York, Flushing, New York 11367, USA
}

\begin{abstract}
The paper deals with optical excitations arising in a one-dimensional chain of identical spheres due optical coupling of whispering gallery modes (WGM). The band structure of these excitations depends significantly on the inter-mixing between WGMs characterized by different values of angular quantum number, $l$. We develop a general theory of the photonic band structure of these excitations taking these effects into account and applied it to several cases of recent experimental interest. In the case of bands originating from WQMs with the angular quantum number of the same parity, the calculated dispersion laws are in good qualitative agreement with recent experiment results. Bands resulting from hybridization of excitations resulting from whispering gallery modes with different parity of $l$ exhibits anomalous dispersion properties characterized by a gap in the allowed values of wave numbers and divergence of group velocity.
\end{abstract}

PACS numbers: 42.60.Da, 42.82.Et, 42.70.Qs 


\section{INTRODUCTION}

Recent proposal of coupled resonator optical waveguides and optical filters $\frac{1,2}{2}$ stimulated interest in systems of optically coupled micro-spheres. It has been known for a long time that electromagnetic modes of an individual sphere (Mie resonances) with large enough values of their angular momentum can have very long radiative life-times ${ }^{3}$. These are so called whispering gallery modes (WHM) characterized by the concentration of the electromagnetic field along the surface of a sphere with an evanescent tail escaping outside. Such field configuration makes it possible to optically couple two spheres positioned in the proximity of one another. Initial work on the optical coupling of the micro-spheres was concentrated on the case of just two spheres (the arrangement is known as photonic atoms or molecules), where the splitting of the modes and the formation of the coupled states was observed $4,5,6$. Recently, however, an interest has shifted toward linear chain of many spheres, which are envisioned as building blocks of various photonic devices, such as waveguides ${ }^{7}$ or delay lines ${ }^{8}$. The obvious result of optical coupling in this system is formation of collective optical excitations propagating along the chain, which we will call supermodes in order to distinguish them from the modes of individual spheres. The supermodes form photonic bands, which were observed by several research groups almost at the same time ${ }^{7.8 .9}$.

One of the popular tools used to analyze experimental results 8 is a simple phenomenological dispersion law of a tight-binding type

$$
\omega=\omega_{0}+\kappa \cos (q d)
$$

where $\omega$ and $\omega_{0}$ are respectively the frequency of the supermode, characterized by a wave number $q$, and that of a single mode WGM resonance; phenomenological coefficient $\kappa$ characterizes the strength of the optical coupling, and $d$ is the period of the structure. This

approach, which was originally suggested in Ref 1 to describe modes of coupled cavities, derive every photonic band from a respective single sphere WGM resonance, characterized by its angular momentum, $l$. Its applicability is based on the assumption that the modes of coupled spheres with different $l$ do not mix, which is certainly not true in the exact sense, but can be approximately valid or not valid at all depending upon the type of modes under consideration.

The admixture of WGM with different angular momentums arises already for two interacting spheres, and results from the violation of spherical symmetry in such systems. 
It has been realized early on that the inter-mode coupling is primary responsible for radiative decay of the coupled modes ${ }^{10}$, and may also affect positions of the resonances in a bi-spherical structure. We will show in this paper that these effects are even more important, and in certain situations, crucial, for the supermodes of the multi-spherical chains. In these systems, instead of individual resonances, one has to consider mixing between bands of collective propagating excitations. Using analogy with solid state physics one can call this phenomenon band-mixing or band hybridization. In this paper, we present a theory of the inter-band coupling in linear chain of spheres based on the tight-binding approach to the optical coupling. This approach was originally formulated in Ref.11, and carefully analyzed for the case of bi-spheres in Ref.12.

Our main goal in this paper is to extend the analysis of Ref 12 to a linear chain of spheres, and apply it to the problem of photonic band structure and dispersion laws of the quasi-stationary (long-living) collective optical excitations of the chain. Using a perturbative approach, we derive analytically dispersion equations characterizing these excitations and analyze them in several particular cases of recent experimental interest. We show, in particular, that in spite of a formal analogy between optical and electronic tight-binding models, the photonic band mixing may lead to very unusual effects, which do not have counterparts in solid state systems.

\section{TIGHT-BINDING MODEL FOR A LINEAR CHAIN OF DIELECTRIC SPHERES}

The system, which will be considered in the paper consists of a number of identical spheres with radius $R$ and refractive index $n$, whose centers are all aligned along the same line (see Fig (1) at a distance $d \geq 2 R$ from each other. We will be interested in collective excitations of this chain, which in the spirit of tight-binding approach is described as a combination of modes of individual spheres. It is pertinent, therefore, to start with a brief review of the properties of a single sphere. Electromagnetic field in this case can be described by vector spherical harmonics (VSH), whose angular dependence is specified by angular number $l$ (angular momentum) and azimuthal number $m$ (z-component of the angular momentum). The radial part of the VSH, $f_{l}(k r)$ is given either by spherical Hankel function of the first kind, $h_{l}^{(1)}(k r)$, for points outside of the sphere or by spherical Bessel function, $j_{l}(n k r)$, for 


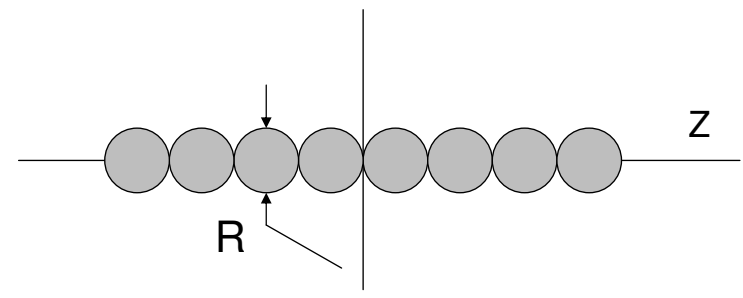

FIG. 1: The chain of spheres of radius $R$ with $Z$-axis along the axis of the chain

points inside the sphere. Parameter $k$ here represents vacuum wave number, $k=\omega / c$, where $\omega$ and $c$ are frequency and the speed of light in vacuum. We will use notation $\mathbf{N}_{m, l}(\mathbf{r})$ and $\mathbf{M}_{m, l}(\mathbf{r})$ for VSH describing electromagnetic waves of TE and TM polarizations respectively. Explicit expressions for functions $\mathbf{N}_{m, l}(\mathbf{r})$ and $\mathbf{M}_{m, l}(\mathbf{r})$ can be found in many sources (see, for instance, Ref 13 ) thus we do not present it here. An isolated dielectric sphere is an open system, therefore, it is more prudent to describe its properties in terms of resonances rather than normal modes. To this end one presents an incident, $\mathbf{E}_{\mathbf{i n c}}$, and scattered by the sphere, $\mathbf{E}_{\mathbf{s}}$, waves in terms of $\mathrm{VSH}$ :

$$
\begin{aligned}
\mathbf{E}_{\mathbf{i n c}} & =\sum_{l, m}\left[\zeta_{l, m} \mathbf{N}_{m, l}(\mathbf{r})+\eta_{l, m} \mathbf{M}_{m, l}(\mathbf{r})\right] \\
\mathbf{E}_{\mathbf{s}} & =\sum_{l, m}\left[a_{l, m} \mathbf{N}_{m, l}(\mathbf{r})+b_{l, m} \mathbf{M}_{m, l}(\mathbf{r})\right]
\end{aligned}
$$

Using Maxwell boundary conditions one establishes relations between coefficients $a_{l, m}$ and $b_{l, m}$ of Eq.(3) and coefficients $\zeta_{l, m}$ and $\eta_{l, m}$ of Eq.(2) in the following form:

$$
\begin{aligned}
& a_{l, m}=\alpha_{l, m} \zeta_{l, m} \\
& b_{l, m}=\beta_{l, m} \eta_{l, m},
\end{aligned}
$$

where scattering coefficients $\alpha$ and $\beta$ are given by

$$
\begin{aligned}
\alpha_{l, m} & =\frac{n^{2} j_{l, m}(n x)\left[x j_{l, m}(x)\right]^{\prime}-j_{l, m}(x)\left[n x j_{l, m}(n x)\right]^{\prime}}{n^{2} j_{l, m}(n x)\left[x h_{l, m}^{(1)}(x)\right]^{\prime}-h_{l, m}^{(1)}(x)\left[n x j_{l, m}(n x)\right]^{\prime}} \\
\beta_{l, m} & =\frac{j_{l, m}(n x)\left[x j_{l, m}(x)\right]^{\prime}-n^{2} j_{l, m}(x)\left[n x j_{l, m}(n x)\right]^{\prime}}{j_{l, m}(n x)\left[x h_{l, m}^{(1)}(x)\right]^{\prime}-n^{2} h_{l, m}^{(1)}(x)\left[n x j_{l, m}(n x)\right]^{\prime}}
\end{aligned}
$$

Here we introduced the dimensionless parameter $x$ defined as $x=k R ; f^{\prime}(x)$ means a derivative of $f$ with respect to $x$. In what follows we will use $x$ as a dimensionless frequency. The coefficients $\alpha$ and $\beta$ as functions of $x$ possess poles on a complex plane. The real parts 
of these poles give frequencies of the respective scattering resonances, while the imaginary parts determine the width of the resonances. It is customary to characterize the latter using a quality factor $Q$ defined as $Q=\operatorname{Re} x / \operatorname{Im} x$. The resonances are characterized by polarization (TE or TM, depending on their origin from $\alpha$ or $\beta$ coefficients, respectively), and two indices, $l$, and $s$, where the former is the respective angular momentum, while the latter is a radial quantum number enumerating poles of $\alpha$ and $\beta$ with the same values of $l$, but different radial distribution of the field (the resonances are degenerate with respect to index $m$ ). Following Ref.12 we will label resonances as lTEs or lTMs. Q-factor of these resonances strongly depends on $l$ reaching very high (up to $10^{9}$ ) values for large enough $l^{3}$. At the same time, for modes with larger $s Q$ decreases, sometimes by an order of magnitude.

The resonances with high $Q$ values correspond to so called whispering gallery modes (WGM), and can be sensibly described as almost stationary (long-living) normal modes of a sphere. The collectivization of these modes in a chain of spheres is the main focus of this paper. The electric field of these modes has an evanescent character in the vicinity of the surface of their respective spheres making the tight-binding description of the optical coupling between adjacent spheres rather accurate $\mathrm{I2}^{\underline{12}}$. This description is based on generalizing the expansions for scattered wave given by Eq. (3) to the case of multiple spheres:

$$
\mathbf{E}_{\mathbf{s}}=\sum_{i, l, m}\left[a_{l, m}^{i} \mathbf{N}_{m, l}\left(\mathbf{r}-\mathbf{r}_{i}\right)+b_{l, m}^{i} \mathbf{M}_{m, l}\left(\mathbf{r}-\mathbf{r}_{i}\right)\right]
$$

where $\mathbf{r}_{i}$ is the position vector of the center of $i$-th sphere. The problem is, however, that VSH in this expansion are defined in different coordinate systems associated with the center of each sphere. In order to apply boundary conditions one need to rewrite them in a common coordinate system. This is achieved with the help of addition theorem $\stackrel{14.15}{ }$, which presents VSH centered at point $\mathbf{r}_{j}$ in terms of VSH centered at a point $\mathbf{r}_{i}$ :

$$
\begin{array}{r}
\mathbf{N}_{l, m}\left(\mathbf{r}-\mathbf{r}_{j}\right)=\sum_{l^{\prime}=1}^{\infty} \sum_{m^{\prime}=-l^{\prime}}^{l^{\prime}}\left[A_{l, m}^{l^{\prime}, m^{\prime}}\left(\mathbf{r}_{j}-\mathbf{r}_{i}\right) \mathbf{N}_{l^{\prime}, m^{\prime}}\left(\mathbf{r}-\mathbf{r}_{i}\right)+B_{l, m}^{l^{\prime}, m^{\prime}}\left(\mathbf{r}_{j}-\mathbf{r}_{i}\right) \mathbf{M}_{l^{\prime}, m^{\prime}}\left(\mathbf{r}-\mathbf{r}_{i}\right)\right] \\
\mathbf{M}_{l, m}\left(\mathbf{r}-\mathbf{r}_{j}\right)=\sum_{l^{\prime}=1}^{\infty} \sum_{m^{\prime}=-l^{\prime}}^{l^{\prime}}\left[A_{l, m}^{l^{\prime}, m^{\prime}}\left(\mathbf{r}_{j}-\mathbf{r}_{i}\right) \mathbf{M}_{l^{\prime}, m^{\prime}}\left(\mathbf{r}-\mathbf{r}_{i}\right)+B_{l, m}^{l^{\prime}, m^{\prime}}\left(\mathbf{r}_{j}-\mathbf{r}_{i}\right) \mathbf{N}_{l^{\prime}, m^{\prime}}\left(\mathbf{r}-\mathbf{r}_{i}\right)\right],
\end{array}
$$

where $A_{l^{\prime}, m^{\prime}}^{l, m}\left(\mathbf{r}_{j}-\mathbf{r}_{i}\right)$ and $B_{l^{\prime}, m^{\prime}}^{l, m}\left(\mathbf{r}_{j}-\mathbf{r}_{i}\right)$ are the so- called translation coefficients, which depends on the mutual position of the spheres. Expressions for the translation coefficients can be found, for instance, in Ref.10 or Ref.12. The latter paper gives a rather detailed 
description of methods for computing these coefficients, which is not an easy computational task, since for modes with large $l$ it involves factorials of very large numbers. Using the addition theorem one can derive a system of equations for the expansion coefficients $a_{l, m}^{i}$ and $b_{l, m}^{i} \underline{10}$

$$
\begin{aligned}
& a_{l, m}^{i}=\alpha_{l, m}\left\{\zeta_{l, m}+\sum_{j \neq i} \sum_{l^{\prime}, m^{\prime}}\left[a_{l^{\prime}, m^{\prime}}^{j} A_{l, m}^{l^{\prime}, m^{\prime}}\left(\mathbf{r}_{j}-\mathbf{r}_{i}\right)+b_{l^{\prime}, m^{\prime}}^{j} B_{l, m}^{l^{\prime}, m^{\prime}}\left(\mathbf{r}_{j}-\mathbf{r}_{i}\right)\right]\right\} \\
& b_{l, m}^{i}=\beta_{l, m}\left\{\eta_{l, m}+\sum_{j \neq i} \sum_{l^{\prime}, m^{\prime}}\left[b_{l^{\prime}, m^{\prime}}^{j} A_{l, m}^{l^{\prime}, m^{\prime}}\left(\mathbf{r}_{j}-\mathbf{r}_{i}\right)+a_{l^{\prime}, m^{\prime}}^{l} B_{l, m}^{l^{\prime}, m^{\prime}}\left(\mathbf{r}_{j}-\mathbf{r}_{i}\right)\right]\right\}
\end{aligned}
$$

Indexes $i$ and $j$ in these equations enumerate spheres, while $l, l^{\prime}, m, m^{\prime}$ correspond to different resonances of the individual spheres. The structure of these equations can be understood by noting that it is equivalent to Eq. (4) and (5), in which the incident field, characterized by coefficients $\zeta_{l, m}$ and $\eta_{l, m}$ is replaced by its sum with the field scattered by all other spheres. This system of equations is valid for an arbitrary collection of spheres, as indicated in Ref 10. For a linear chain, in which all position vectors $\mathbf{r}_{i}$ can be chosen parallel to each other and to the axis of the chain, these equations can be simplified. If one chooses the $Z$-axis of the coordinate system along the chain, the translation coefficients can be shown to become $A_{l, m}^{l^{\prime}, m^{\prime}}=A_{l}^{l^{\prime}} \delta_{m, m^{\prime}}$ and $B_{l, m}^{l^{\prime}, m^{\prime}}=B_{l}^{l^{\prime}} \delta_{m, m^{\prime}}$. Thus, the sum over $m^{\prime}$ in Eq. (10) and (11) disappears, making the component of the angular momentum along the axis of the chain a conserving quantity. This fact obviously reflects the axial symmetry of this system. In what follows, we, for concreteness, shall assume that $m=1$; results for other values of $m$ can be obtained from our general formulas by recalculating parameters $\alpha$ and $\beta$ as well as the translational coefficients. Thus, we can abridge our notations by dropping index $m$ all together. At this point we also omit the terms $\eta_{l, m}$ and $\zeta_{l, m}$ describing the external incident wave, which leaves us with a system of homogeneous linear equations and the problem of finding their normal modes instead of the problem of scattering of an external wave.

Thus, we present the equations for the scattering coefficients in the following form:

$$
\begin{aligned}
& a_{l}^{i}=\alpha_{l} \sum_{j \neq i} \sum_{l^{\prime}}\left[a_{l^{\prime}}^{j} A_{l}^{l^{\prime}}(i, j)+b_{l^{\prime}}^{l} B_{l}^{l^{\prime}}(i, j)\right] \\
& b_{l}^{i}=\beta_{l} \sum_{j \neq i} \sum_{l^{\prime}}\left[b_{l^{\prime}}^{j} A_{l}^{l^{\prime}}(i, j)+a_{l^{\prime}}^{j} B_{l}^{l^{\prime}}(i, j)\right],
\end{aligned}
$$

where the position vectors, $\mathbf{r}_{i}$, of the spheres in the arguments of the translation coeffi- 
cients are for shortness replaced with sphere's numbers, $i$. Eq.(12) and (13) have been used by Miyazaki and Jimba in Ref.12 for exact numerical analysis of a bi-sphere. They also developed for this system an approximate method of solution of these equations that reproduced results of the exact calculations with a good accuracy. Here we generalize this method for a system with an arbitrary, including infinite, number of spheres, where its use becomes crucial since exact numerical calculations grow increasingly more involved and expensive with the increase in the number of spheres in the chain. We will show here that using the expanded version of the tight-binding approximation we are able to derive analytically dispersion equations describing collective excitations of the chain, which can be solved numerically with minimal computational efforts.

The reduction of Eq.(12) and Eq.(13) to an analytically tractable form is based on several important properties of the translation coefficients, $A_{l}^{l^{\prime}}$, and $B_{l}^{l^{\prime}}$. First of all, in the case of $l \gg 1$ the calculation of these coefficients can be significantly simplified with the help of the so-called maximum term approximation ${ }^{12}$ that allows presenting these coefficients in the following form

$$
\begin{aligned}
& A_{l}^{l^{\prime}}(i, j) \cong-2 l(-1)^{l+1} \sqrt{\frac{l+l^{\prime}}{\pi\left(l^{\prime}+1\right)(l-1)}} \times \frac{l^{l}\left(l^{\prime}\right)^{l^{\prime}}}{\left(l^{\prime}+1\right)^{l^{\prime}+1}(l-1)^{l-1}} h_{l+l^{\prime}}^{(1)}(\eta x|i-j|) \\
& B_{l}^{l^{\prime}}(i, j) \cong i \frac{x|i-j|}{l l^{\prime}} A_{l}^{l^{\prime}}(i, j)
\end{aligned}
$$

where $\eta$ defined as $\eta=\left|\mathbf{r}_{i}-\mathbf{r}_{j}\right| / R \geq 2$ is a dimensionless distance between the centers of the spheres. In the case of spheres touching each other, which we shall assume in our numerical calculations, $\eta=2$. Taking into account properties of the Hankel function $h_{l}^{(1)}(x)$ in Eq.(14), one can see that the translation coefficients quickly decrease with the distance between the spheres. This property, which is a manifestation of the evanescent nature of the optical coupling between the spheres, allows one to keep in the sum over the spheres in Eq.(12) and (13) only terms with $j=i \pm 1$. The resulting equations constitute the nearest neighbors approximation for the chain of the spheres. The sum over $l^{\prime}$ describes coupling between supermodes with different angular modes, which is the main subject of the present paper. These equations also contain terms proportional to translation coefficients $B_{l}^{l^{\prime}}$, which are responsible for coupling between supermodes with different polarizations. It follows, however, from Eq. (15) that for the nearest neighbors $B_{l}^{l^{\prime}} \ll A_{l}^{l^{\prime}}$ for $l, l^{\prime} \gg 1$. We will see later that the main contribution to the sum over $l^{\prime}$ comes mostly from $l^{\prime} \lesssim l$, and the 
cross-polarization coupling can, therefore, be neglected in most cases. In what follows we will also discard terms $\zeta_{l}$ and $\eta_{l}$ responsible for external excitation. This leaves us with a system of homogeneous linear equations and the problem of finding their normal modes instead of the problem of scattering of an external wave. Thus, we present the final tight-binding equations describing $T E$ and $T M$ supermodes as

$$
\begin{aligned}
& \frac{1}{\alpha_{l}} a_{l}^{i}=\sum_{l^{\prime}} A_{l}^{l^{\prime}}\left(a_{l^{\prime}}^{i-1}+a_{l^{\prime}}^{i+1}\right) \\
& \frac{1}{\beta_{l}} b_{l}^{i}=\sum_{l^{\prime}} A_{l}^{l^{\prime}}\left(b_{l^{\prime}}^{i-1}+b_{l^{\prime}}^{i+1}\right),
\end{aligned}
$$

where the translation coefficients $A_{l}^{l^{\prime}}=A_{l}^{l^{\prime}}(i, i+1)$ play the role of the inter-site coupling parameters of the tight-binding approximation. They also play an important additional role of being coupling coefficients for supermodes with different $l$. These equations are very similar to electronic tight binding equations describing, for instance, s-p hybridization ${ }^{16,17}$. There is, however, a significant difference between optical and electronic problems caused by the non-Hermitian nature of matrix $A_{l}^{l^{\prime}}$ describing the coupling in the optical case. We will see below that this peculiarity of optical tight-binding equations is responsible for the anomalous dispersion properties of the system under consideration. The non-Hermitian nature of the optical band coupling appears also in a multiple scattering (optical KorringaKohn-Rostocker) approach to this problem developed in Ref.18.

\section{COLLECTIVE EXCITATIONS IN THE LINEAR CHAIN OF MICRO- SPHERES}

\section{A. Single band approximation}

Neglecting terms with $l^{\prime} \neq l$ in equations (16) and (17) one obtains a simple single-band nearest-neighbor interaction model describing bands of the collective supermodes originating

from $l T E s$ or $l T M s$ WGM's of an individual sphere with frequencies $x_{l s}$. For a TE band, for instance, one has

$$
\frac{1}{\alpha_{l}} a_{l}^{i}=A_{l}^{l}\left(a_{l}^{i-1}+a_{l}^{i+1}\right)
$$

and the equation for TM bands has the same form with an obvious substitution of $\beta_{l}$ instead of $\alpha_{l}$. In order to isolate a single band, and convert this equation into a standard 

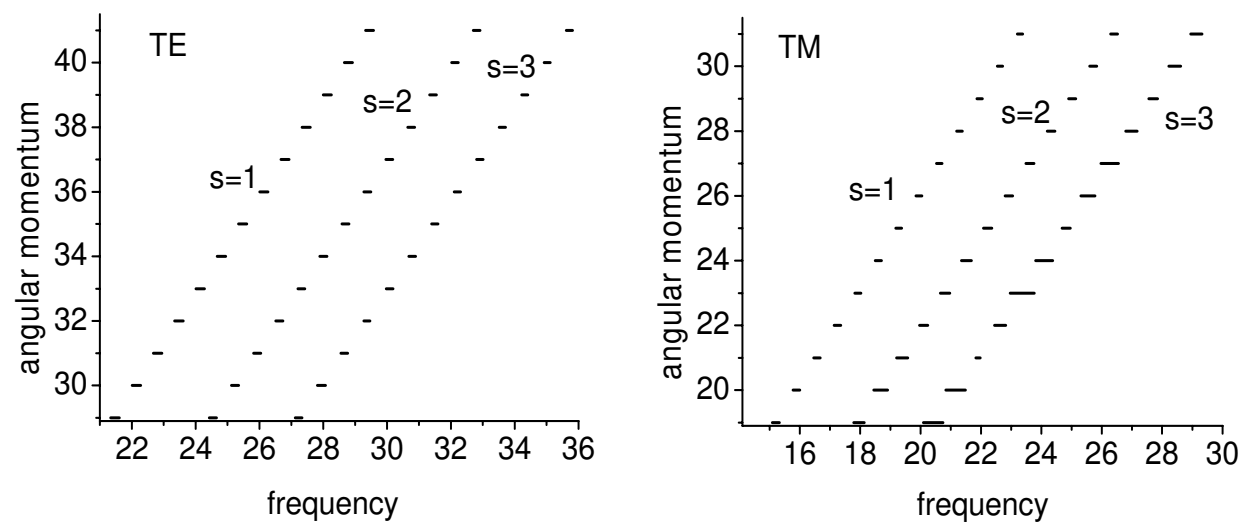

FIG. 2: Positions and widths of several TE and TM supermodes obtained in a single band approximation.

tight-binding form, we can take advantage of the fact that the expected width of the band of the $l, s$ supermode, $\delta x_{l s}$, is rather small, $\delta x_{l s} \ll x_{l s}$. In this case we can expand the single sphere scattering parameters $\alpha$ or $\beta$ around their respective resonance frequencies $x_{l s, T E}$ or $x_{l s, T M}$. Taking into account the resonant nature of the scattering parameters, we can write down:

$$
\frac{1}{\alpha_{l}} \approx \frac{\left(x-x_{l s}+i \gamma_{l s}\right)}{i \Delta_{l s}},
$$

and the similar equation for the TM polarization. Parameters $\Delta_{l s}$ in Eq.(19) are real valued positive quantities for all $l$ and $s^{12}$, and $\gamma_{l s}$ represents the rate of the radiative decay of the respective WGM. Taking into account that translation parameters $A_{l}^{l^{\prime}}$ in the maximum term approximation are almost purely imaginary quantities:

$$
A_{l}^{l^{\prime}} \cong i(-1)^{l+1}\left|A_{l}^{l^{\prime}}\right|
$$

and calculating them at the resonance frequencies $x_{l s}$, one can re-write Eq.(18) or similar equation for the TM polarization in the standard for the tight-binding approximation form

$$
\left(x-x_{l s}+i \gamma_{l s}\right) a_{l}^{i}=(-1)^{l} \Delta_{l s} \tilde{A}_{l s}\left(a_{l}^{i-1}+a_{l}^{i+1}\right),
$$

where $\tilde{A}_{l s}=\left|A_{l}^{l}\left(x_{l s}\right)\right|$. Normal modes of this system of equations are harmonic waves

$$
a_{l}^{i} \propto \exp \left(i q_{l s} z_{i}\right)
$$

where $z_{i}$ is the $z$ coordinate of the $i$-th sphere, and $q_{l s}(x)$ is a Bloch wave number. It satisfies the dispersion equation of the same type as Eq.(1), where we can now give a microscopic 
expression for the phenomenological coupling parameter $\kappa$ :

$$
\kappa_{l s}=(-1)^{l} 2 \Delta_{l s} \tilde{A}_{l s}
$$

Dispersion equation (Eq.(11) ) describes a band of excitations, which is symmetric with respect to the WPG frequency $x_{l s}$, and whose boundaries $x_{b}^{ \pm}$are given by expression

$$
x_{b}^{ \pm}=x_{l s} \pm \kappa_{l s}
$$

In the case of a chain consisting of a finite number of spheres, $N$, the dispersion equation (11) can be used to find the spectrum of the respective frequencies. To this end, one has to impose obvious boundary conditions for the coefficients $a_{l}^{i}$, which read as $a_{l}^{0}=a_{l}^{N+1}=0$, where we assume that first sphere in the chain is assigned number $i=1$. These boundary conditions determine the allowed values of the Bloch wave number $q_{l s}: q_{l s} \eta=\pi n /(N+1)$, where $n$ changes from 1 to $N$. Applying this result to the case of only two spheres we find that there are two possible values of $q_{l s}$, namely $q_{l s} \eta=\pi / 3$ and $q_{l s} \eta=2 \pi / 3$. The respective values of frequencies $x_{l s}^{1,2}=x_{l s} \pm \kappa_{l s} / 2$, reproduce the results of Ref.12 obtained for the case of a bi-sphere in the single mode approximation. The radiative decay characterized by $\gamma_{l s}$ makes the supermodes quasi-stationary; obviously the whole concept of the collective excitations with different $q$ can only make sense if $\gamma_{l s} \ll \kappa_{l s}$. Calculations as well as experiments 7.8 .9 show that for modes with large $l$ this inequality is well satisfied.

This simple picture could describe supermodes that do not overlap spectrally since only in this case omitting terms with $l^{\prime} \neq l$ in Eq.(16) can be justified. The bands of collective excitations in the chain of microspheres, however, almost certainly overlap with at least one or more other bands. In Fig 2 we present positions and widths of a number of supermode bands calculated in the single band approximation. It should be understood, however, that because of the inherently present radiative decay, the concept of allowed and forbidden bands is not very well defined even for modes with relatively large Q-factors. Nevertheless, we will use these terms to describe spectral regions, where the wave number $q$ would have been purely real or purely imaginary in the absence of the decay, respectively. In reality, the wave number is a complex valued quantity at all real frequencies, and a difference between allowed and forbidden bands manifests itself only in relative magnitudes of real and imaginary parts of $q$ : $\operatorname{Im} q \ll \operatorname{Re} q$ within the allowed band, and $\operatorname{Im} q \gg \operatorname{Re} q(\bmod \pi)$ within the forbidden bands. In the vicinity of the band boundaries, $x_{b}^{ \pm}$, the real and imaginary parts are of the same order: $\operatorname{Im} q \simeq \operatorname{Re} q$. 


\section{B. Inter-band coupling and band hybridization in the linear chain of spheres}

In this subsection we will take into account non-diagonal terms in Eq.(16) and (17), which are responsible for coupling between bands with different angular momentum described in the previous subsection. Since calculations for TE and TM modes are almost identical, we shall consider explicitly only TE polarization; results for TM polarization, which we will use for numerical examples, can be easily obtained with the help of obvious substitution of the parameters.

A particular solution to the system of equations (16) can be easily found in the form of a harmonic wave: $a_{l}^{(i)}=a_{l} e^{i q z_{i}}$, where $a_{l}$ satisfy the following algebraic equations:

$$
\frac{1}{\alpha_{l}} a_{l}+2 \cos (q \eta) \sum_{l^{\prime}} A_{l}^{l^{\prime}} a_{l^{\prime}}=0
$$

Let us consider this equation in a frequency region occupied by a single-band supermode $l_{0} T E s_{0}$ characterized by quantum numbers $l=l_{0}$ and $s=s_{0}$. We are interested in finding corrections to the single band dispersion law for this supermode caused by its interaction with other bands. In order to develop an approximate analytical solution for this problem we have to take into account several circumstances. First, main corrections to the diagonal approximation come from those $l$, which correspond to bands spectrally overlapping with $l_{0} T E s_{0}$. It should be remembered, however, that the bands discussed in the previous subsection are broadened because of the radiative decay, and the term overlap should be understood with this fact in mind. One can see from Fig 2 that all supermodes with $l>l_{0}$ are spectrally well separated from $l_{0} T E s_{0}$, thus the respective terms in Eq.(16) can be considered a small perturbation despite the fact that $A_{l_{0}}^{l}>A_{l}^{l}$ for $l>l_{0}$. Terms with $l<l_{0}$ are more important since according to Fig 2 respective bands can be spectrally in close proximity to $l_{0} T E s_{0}$,

or even overlap with it. On the other hand coupling coefficients $A_{l_{0}}^{l}$ quickly decrease when $l$ becomes smaller than $l_{0}$. Thus, the effect of coupling to these supermodes depends on an interplay between resonant enhancement due to spectral proximity, and decrease in the coupling parameter $A_{l_{0}}^{l}$.

In most practical situation, among all the bands contributing to the sum over $l^{\prime}$ there is just one, which we will label as $l_{1} T E s_{1}$, effecting the supermode under consideration in a most significant way. Usually the interaction with such a band is too strong to allow for a perturbative treatment. Following the terminology of Ref 12 we will call $l_{0} T E s_{0}$ and $l_{1} T E s_{1}$ 
significant supermodes, while the remaining ones are called bath or reservoir bands and can be treated perturbationally. We will show here that in the system under consideration the inter-band coupling can result in three qualitatively different types of the modifications of the dispersion laws. One, which is characteristic for weaker interaction can be called band renormalization, while the others, requiring a stronger coupling, are more appropriately called band hybridization. There might be two types of the hybridization, one arising, when the interacting bands are characterized by angular momentum indexes of the same parity, and the other one corresponding to the situation, when they have angular momentum indexes of different parities. We will show that the hybrid band arising in each of these cases exhibit qualitatively different dispersion properties.

We will begin by developing a general theory capable of describing all possible situations, and then consider conditions controlling a transition from one regime to the other. As it was mentioned before, the interaction between $l_{0} T E s_{0}$ and $l_{1} T E s_{1}$ should be taken into account exactly, while the contribution from all other supermodes can be treated perturbatively. Accordingly, we present the system of Eq.(25) for the amplitudes of the supermodes in the following form:

$$
\begin{aligned}
& {\left[\frac{1}{\alpha_{l_{0}}}+2 A_{l_{0}}^{l_{0}} \cos (q \eta)\right] a_{l_{0}}+2 \cos (q \eta) A_{l_{0}}^{l_{1}} a_{l_{1}}+2 \cos (q \eta) \sum_{l \neq l_{0}, l_{1}} A_{l_{0}}^{l} a_{l}=0} \\
& {\left[\frac{1}{\alpha_{l_{1}}}+2 A_{l_{1}}^{l_{1}} \cos (q \eta)\right] a_{l_{1}}+2 \cos (q \eta) A_{l_{1}}^{l^{0}} a_{l^{0}}+2 \cos (q \eta) \sum_{l \neq l_{0}, l_{1}} A_{l_{1}}^{l} a_{l}=0} \\
& {\left[\frac{1}{\alpha_{l}}+2 A_{l}^{l} \cos (q \eta)\right] a_{l}+2 \cos (q \eta) \sum_{l^{\prime} \neq l} A_{l}^{l^{\prime}} a_{l^{\prime}}=0 .}
\end{aligned}
$$

where in the first two lines we extracted from the sum over $l$ the terms with $l=l_{0}$ and $l=l_{1}$, and wrote down the separate equations for the respective amplitudes. The last line in Eq. (26) represents the equation for the reservoir modes, which we solve for $a_{l}$ and substitute the solution to the first two lines of this equation. In the resulting double sum over $l$ and $l^{\prime}$, we keep only terms with $l^{\prime}=l_{0}$ and $l^{\prime}=l_{1}$. This procedure, which constitutes the second order perturbation theory for the reservoir bands, results in a system of equations for the amplitudes of the significant supermodes, which can be presented in the following form:

$$
\begin{aligned}
& {\left[\frac{1}{\alpha_{l_{0}}}+2 \tilde{A}_{l_{0}}^{l_{0}} \cos (q \eta)\right] a_{l_{0}}+2 \cos (q \eta) \tilde{A}_{l_{0}}^{l_{1}} a_{l_{1}}=0} \\
& {\left[\frac{1}{\alpha_{l_{1}}}+2 \tilde{A}_{l_{1}}^{l_{1}} \cos (q \eta)\right] a_{l_{1}}+2 \cos (q \eta) \tilde{A}_{l_{1}}^{l^{0}} a_{l^{0}}=0}
\end{aligned}
$$


The weak interaction with the reservoir results here in the renormalization of the coupling coefficients according to the rule:

$$
A_{l}^{l^{\prime}} \rightarrow \tilde{A}_{l}^{l^{\prime}}=A_{l}^{l^{\prime}}\left[1+\cos (q \eta) \sum_{\nu \neq l, l^{\prime}} \frac{\Omega_{l \nu}^{l^{\prime}}}{\cos (q \eta)-\cos \left(q_{\nu} \eta\right)}\right]
$$

where

$$
\Omega_{l \nu}^{l^{\prime}}=\frac{A_{l}^{\nu} A_{\nu}^{l^{\prime}}}{A_{l}^{l \prime} A_{\nu}^{\nu}}
$$

is the inter-band interaction constant, and $\cos \left(q_{\nu} \eta\right)$ corresponds to the single band approximation for the reservoir modes defined as

$$
\cos \left(q_{\nu} \eta\right)=-\frac{1}{2 \alpha_{\nu} A_{\nu}^{\nu}}
$$

In contrast to Sec $\amalg$ we have omitted here subindex $s$ in our notations for wave numbers $q_{\nu}$. The reason for this is that there is no summation over $s$ in any of the equations describing the inter-supermode coupling, such as Eq. (25) or (26) , and the dependence on this index appears in an explicit form only if we expand scattering coefficients $\alpha_{l}$ around a respective WGM. Unlike Sec $\amalg$ we cannot do this here, because we take into account contributions from bands in the frequency region, which can be relatively far away from their respective parent WGMs. Thus, the sum over $\nu$ in Eq. (28) contains contributions from terms for which the vicinities of $x_{l_{0} s_{0}}$ and $x_{l_{1} s_{1}}$ belong to the forbidden bands of respective supermodes, so that their (mostly imaginary) parameters $q_{\nu}$, defined by Eq. (30), can no longer be associated with a particular WGM.

Eq.(27) describes new bands formed from the initial $l_{0} T E s_{0}$ and $l_{1} T E s_{1}$ bands. In order to simplify notations we will omit for now the angular momentum indices, and will label these new bands simply as $q_{+}$and $q_{-}$. We can present dispersion equations for each of $q_{ \pm}$ in the form:

$$
\begin{aligned}
\cos \left(q_{ \pm} \eta\right) & =\frac{1}{2}\left(\frac{1}{1-\tilde{\Omega}_{l_{0} l_{1}}}\right) \times \\
& {\left[\cos \left(\tilde{q}_{l_{0}} \eta\right)+\cos \left(\tilde{q}_{l_{1}} \eta\right) \pm \sqrt{\left[\cos \left(\tilde{q}_{l_{0}} \eta\right)-\cos \left(\tilde{q}_{l_{1}} \eta\right)\right]^{2}+4 \tilde{\Omega}_{l_{0} l_{1}} \cos \left(\tilde{q}_{l_{0}} \eta\right) \cos \left(\tilde{q}_{l_{1}} \eta\right)}\right] }
\end{aligned}
$$

where $\tilde{\Omega}_{l_{0} l_{1}}$ is defined by Eq.(29), in which we set $l^{\prime}=l=l_{0}, \nu=l_{1}$, drop the upper index as duplicate, and replace the coupling coefficients with their renormalized according 
to Eq.(28) values. Similarly, $\tilde{q}_{l}$ are defined as in Eq.(30), but also with renormalized coupling coefficients.

Eq.(31) can be considered either as equations for wave numbers $q_{ \pm}$as functions of frequency $x$, or as equations for two frequencies $x_{ \pm}$as functions of wave number $q$. The choice depends on an experimental situation under consideration. In the transport experiments of the type carried out in Ref. 7 or 9 the frequency is fixed by an external excitation, and $q$ is determined from the experiment. In this case we have to solve this equation for $q_{ \pm}$treating $x$ as an independent real valued variable. The resulting wave numbers are complex, and their imaginary parts characterize the spatial decay of the wave along the chain. On the other hand, in the resonance experiments of Ref. 8 the wave number is fixed, and frequency is measured. In this case, we have to solve our dispersion equation for $x$ considering $q$ as a real quantity. The frequencies obtained as a result contain imaginary parts, which describe the spectral width of the respective resonances. It is important to understand that because the dispersion equation is complex, the functions $\operatorname{Re}\left(q_{ \pm}(x)\right)$ and $\operatorname{Re}\left(x_{ \pm}(q)\right)$ obtained in these two approaches are not inverse of each other. In this paper we will focus on finding $q_{ \pm}(x)$ because it presents greater interest from the stand point of experiment as well as applications.

For two special values of frequency, Eq.(31) can be solved exactly. Indeed, consider $x=x_{l_{0} s_{0}}$, where $\cos \left(\tilde{q}_{l_{0}}\right)$ is exactly equal to zero regardless of the renormalization of the coupling coefficients. In this case the term responsible for the inter-band coupling vanishes, and we obtain that one of the $\cos q_{ \pm}$is also equal to zero. The same is obviously valid for $x=x_{l_{1} s_{1}}$. We can conclude, thus that the centers of all bands, where $q_{ \pm} \eta=\pi / 2$, correspond to frequencies of the respective parent WGM resonances regardless of the presence of the inter-band coupling. In resonant experiments with finite chains ${ }^{8}$ the admissible values of $q$ are determined by the boundary conditions at the ends of the chain. In this situation, the center of the band is accessible only in systems with odd number of spheres. Therefore, spectra of chains with odd and even number of spheres can be distinguished from each other by, respectively, presence or absence of excitations at frequencies corresponding to single sphere whispering gallery modes. This conclusion is in complete agreement with observations of Ref. 8 .

In general case we can solve Eq.(31) numerically by consecutive iterations. The zero iteration corresponds to neglecting the bath-induced renormalization of the coupling coefficients, and produces two zero-order dispersion curves $q_{ \pm}^{(0)}$. At the next step, $q_{ \pm}^{(0)}$ are substituted 
to Eq. (28) and Eq. (30) producing a pair (one for $q_{+}^{(0)}$ and one for $q_{-}^{(0)}$ ) of new values of the coupling coefficients and "single-band" wave numbers $\tilde{q}_{l}$. These new values go back to Eq.(31), one to the "+" version of it, and the other to the "-" version. The procedure can be repeated as many times as necessary to achieve its convergence. The experience shows, however, that a good approximation for $q_{ \pm}$can already be obtained after only the first iteration.

While the contributions from the reservoir bands can be important, the main qualitative characteristics of the dispersion laws of the significant supermodes can be understood from the zero order approximation, which is presented by Eq.(31) without the renormalization of the coupling coefficients. We are focused on the frequency region, where expression $\cos q_{l_{0}}-\cos q_{l_{1}}$ is small because this is where the main effects of the inter-band interaction are expected. The strongest effects occur in the vicinity of a resonance between supermodes $l_{0}$ and $l_{1}$, when

$$
\operatorname{Re}\left[\cos \left(q_{l_{0}} \eta\right)\right]=\operatorname{Re}\left[\cos \left(q_{l_{1}} \eta\right)\right]
$$

if it takes place in the frequency range covering initial $l_{0} T E s_{0}$ and $l_{1} T E s_{1}$ bands. It should be noted, however, that because of the radiative decay the band boundaries particularly for the band with larger $s$ are not that very well defined, and the strong effects can take place even if the resonance condition is fulfilled in the forbidden band of one or even both of the modes, if the resonance point lies in proximity of a band boundary. It is important to remember also that Eq.(32) is not equivalent to $\operatorname{Re}\left(q_{l_{0}}\right)=\operatorname{Re}\left(q_{l_{1}}\right)$, which is often accepted as a resonance condition.

The properties of the dispersion curves in the vicinity of the resonance point are determined by two circumstances: (i) the parity of single band dispersion laws, and (ii) the relation between $\left[\operatorname{Im}\left[\cos \left(q_{l_{1}} \eta\right)\right]-\operatorname{Im}\left[\cos \left(q_{l_{0}} \eta\right)\right]\right]^{2}$ and $\Omega_{l_{0} l_{1}} \cos \left(q_{l_{0}} \eta\right) \cos \left(q_{l_{1}} \eta\right)$, which determines if the band interaction is weak or strong. In most cases the overlapping supermodes originate from WGM with different $s$, and, therefore, usually, one of the imaginary parts in the expression above is significantly (orders of magnitude) larger than the other one. In this case the stronger decaying supermode determines the nature of the modification of the dispersion laws. It is quite obvious that the strong coupling case (band hybridization) would correspond to the interaction terms prevailing over the dissipative terms, and the weak coupling regime (band modification) would take place in the opposite situation. If 
the resonance condition is not fulfilled in the spectral region of interest the modification of the dispersion law always belongs to the weak coupling case. What is surprising, however, is that even in this case the modification can be so strong as to render the perturbative treatment of interaction between the significant modes not very accurate.

An important peculiarity of the dispersion curves described by Eq.(31) distinguishing it from other cases of mode coupling (polaritons, for instance) is the sign of the interband interaction parameter, $\Omega_{l_{0} l_{1}}$. Using properties of the coupling coefficients $A_{l}^{l^{\prime}}$ it is easy to show that this parameter is always positive regardless of the parities of the angular momentum indexes $l$ and $l^{\prime}$. The parity of $l$ according to Eq.(21) determines the sign of the slope of the respective dispersion laws in the vicinity of band boundaries. Taking into account that in the frequency region of interest $\cos \left(q_{l_{0}} \eta\right)$ and $\cos \left(q_{l_{1}} \eta\right)$ are, at least, of the same sign, we conclude that the entire interaction term $\Omega_{l_{0} l_{1}} \cos \left(q_{l_{0}} \eta\right) \cos \left(q_{l_{1}} \eta\right)$ is always positive regardless of the interacting bands having slopes of the same or different signs. We will see in the subsequent sections that this feature of the inter-band interaction, which results from the non-Hermitian nature of the matrix $A_{l}^{l^{\prime}}$, is responsible for a rather unusual form of the dispersion laws describing hybridized bands.

\section{Weak inter-band coupling}

As an example of the band modification we consider dispersion curves emerging as a result of interaction between 29TM1 and 25TM2 supermodes. This choice is motivated by experiments of Ref.8, where these dispersion curves have been measured. One can see from Fig 2 that the bands of these two supermodes slightly overlap. However, considering $\operatorname{Re}\left[\cos \left(q_{29} \eta\right)\right]$ and $\operatorname{Re}\left[\cos \left(q_{25} \eta\right)\right]$ we find that the resonance between these two bands does not occur, thus, we should expect in this case just a modification of the dispersion laws without a significant reconstruction of the band structure.

Fig 3 presents the results obtained after first two iterations of numerical solutions of Eq.(31) for these two modes along with the respective initial bands. The second iteration takes into account all reservoir modes from $l=1$ to $l=40$. One can see that the effect of the reservoir modes is almost zero in the vicinity of the center of the band, but slightly increases toward the edges of the initial bands, which confirms our choice of significant and reservoir modes. However, outside of the allowed bands of the 29TM1 and TM24, 2 modes 


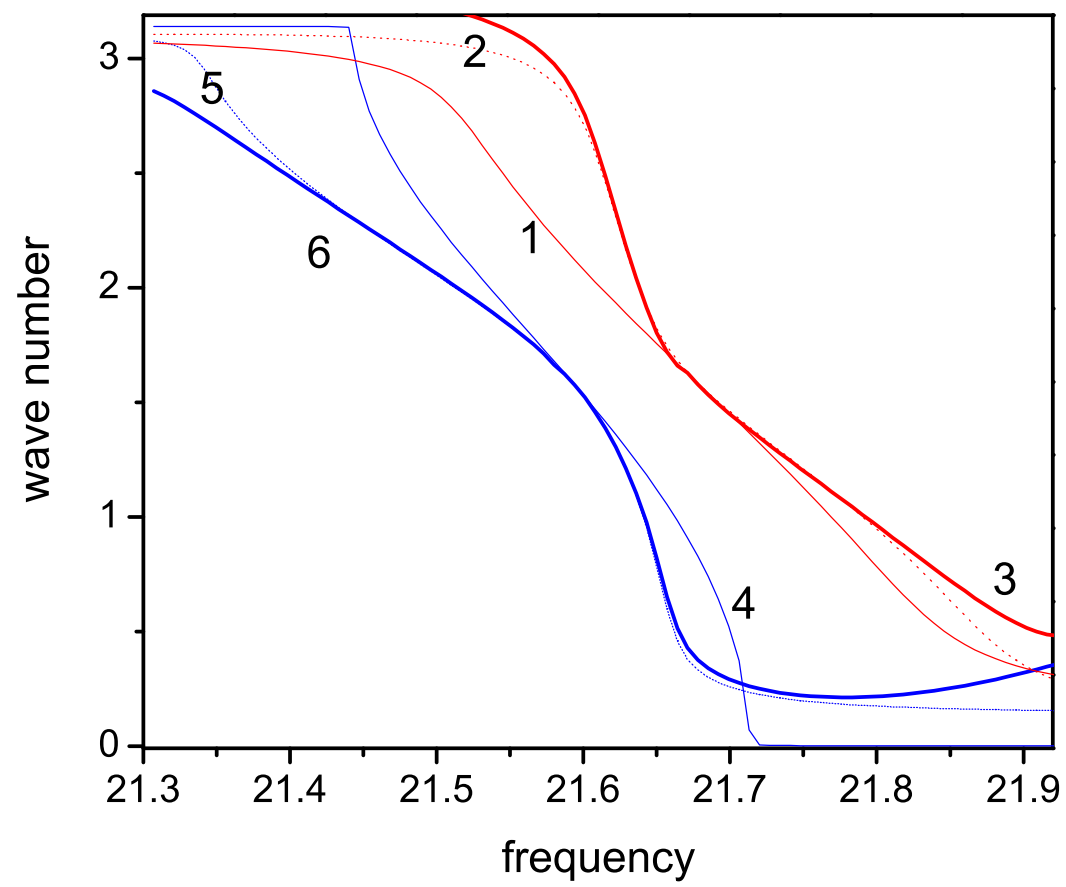

FIG. 3: Dispersion curves of $29 T M 1$ and $24 T M 2$ bands. Dash lines labelled 1 and 4 presents single-band dispersion curves, thin lines labelled 2 and 5 demonstrates dispersion cruves found without reservoir modes taken into account, and finally thick lines labelled 3 and 6 show dispersion curves with reservoir modes included

designation of these modes as significant ones may no longer be valid because we can trespass to the allowed band of one or several of other modes, which we treated here as the bath. It is obvious that in those frequency regions the approximation used to obtain Fig 3 is no longer valid.

The shape of the modified curves can be easily understood on the basis of our general analysis of Eq.(31). As we explained above at $\tilde{q}_{l} \eta=\pi / 2$ modified and initial dispersion laws coincide, but farther away from the center of the respective bands the modified dispersion laws are pushed away from the initial curves. The characteristic shape of the dispersion curves results from the fact that the corrections to the initial dispersion laws due to the inter-band coupling have different signs for 29TM1 and 25TM2 bands: its negative for the former and positive for the latter. The most strong modifications of both curves occur at the 


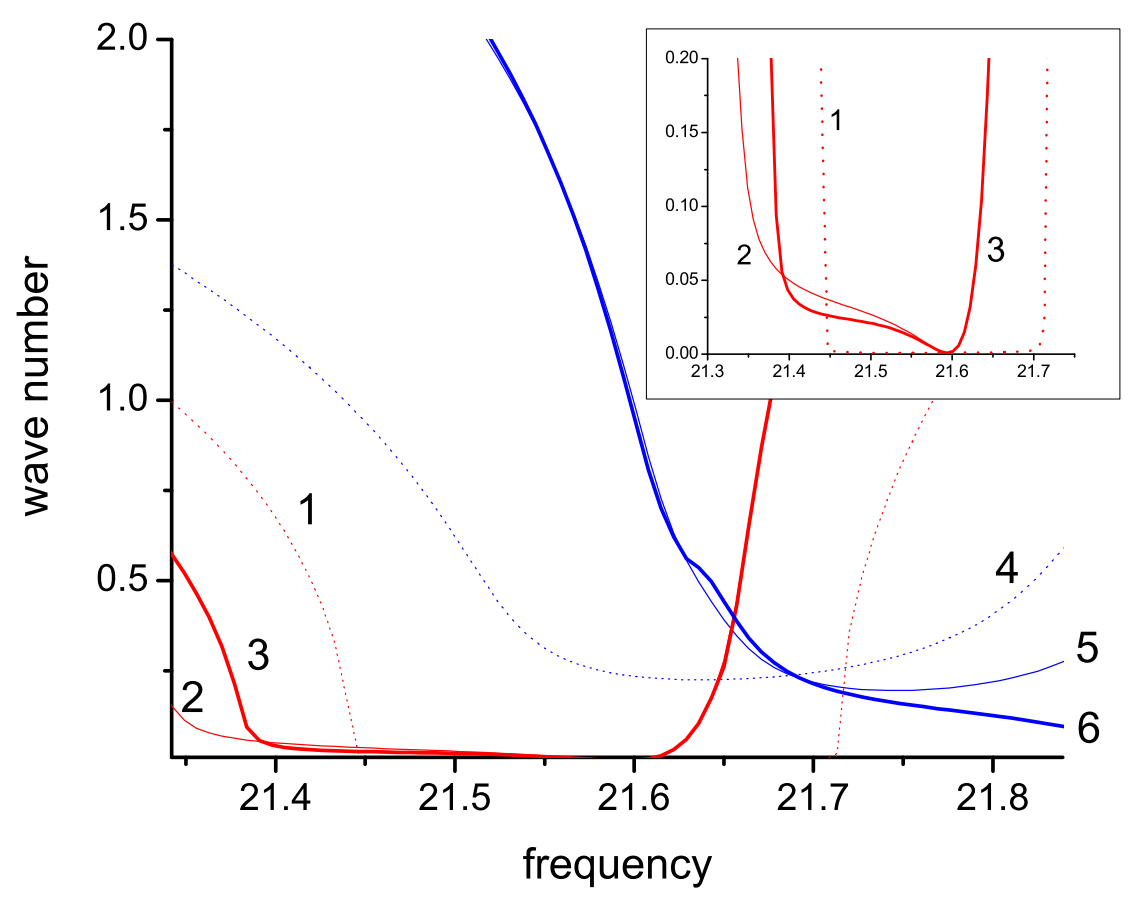

FIG. 4: Decay rates of $29 T M 1(1,2$ and 3) and $24 T M 2(4,5$, and 6$)$ bands. The labelling of the curves is the same as in Fig 3 The insert shows, in a magnified form, the spectral region corresponding to 29TM1 mode. One can see a significant increase in the radiative decay rate of this mode in the vicinity of the new band boundaries, which, however, decreases to the initial WGM value at $x=x_{29,1}$

lower frequency boundaries of their respective bands. This modification can be described as a shift of the boundaries of the respective bands, which is more prominent for $29 T M 1$, whose initial band has much better defined boundaries because of the smaller decay. These boundaries are not very well defined for modified $29 T M 1$ band, however, which tells us about an increase in the radiative decay of this supermode caused by the admixture of $25 T M 2$ band. This increase, however, is not very dramatic, and allows keeping the concept of allowed and forbidden bands as a convenient tool to characterize different spectral regions. The frequency dependence of the decay rates, characterized by $\operatorname{Im} \tilde{q}_{l}$ are shown in Fig 4 , where the positions of the band boundaries characterized by an abrupt change in the decay rate are seen much clearer. Another interesting effect seen in this figure is that the frequency $x_{29 T M 1}$ of the respective WGM is no longer at the center of the modified band. This is, obviously, 
caused by the shift of the band boundaries, which for both low- and high-frequency edges occurs toward lower frequencies. This shift is also responsible for an asymmetry of the band, which manifests itself in the different growth of the decay rates toward left and right boundaries.

We can estimate the shifts of the band boundaries for 29TM1 and 25TM2 supermodes if we neglect the renormalization of the coefficients $A_{l}^{l_{1}}$, and treat the inter-band interaction parameter $\Omega_{l_{1} l_{0}}$ as a small perturbation. Then, employing the approximation for coefficients $\alpha_{l}$ given by Eq.(19), and neglecting the frequency dependence of the coupling coefficients we can find for new band boundaries, $\tilde{x}_{b}^{ \pm}$, defined as frequencies at which $\operatorname{Re}\left[\cos \left(q_{ \pm} \eta\right)\right]=1$ the following expressions

$$
\tilde{x}_{b_{l}}^{ \pm}=x_{b_{l}}^{ \pm} \mp \frac{\Omega_{l \nu} \kappa_{l s}}{1 \mp \cos \left[q_{\nu}\left(x_{b_{l}}^{ \pm}\right) \eta\right]}
$$

Index $l$ in this equation refers to the band whose boundaries we are calculating, while the index $\nu$ signifies its interacting counterpart. Since $\Omega_{l \nu}$ is always positive, and $\kappa_{l s}$ is negative for both participating modes, the sign of the correction is determined by the values of $\cos q$ of the $\nu$-th band at the initial boundaries of the $l$-th band. Since its quite likely that these boundaries lie in the forbidden band of the $\nu$-th band, the values of these cosines are not limited by unity. In the case of $29 T M 1$ supermode interacting with $25 T M 2$ we find that

$$
\begin{aligned}
\cos \left[q_{25}\left(x_{29,1}^{+}\right) \eta\right] & \approx 0.18 & \cos \left[q_{25}\left(x_{29,1}^{-}\right) \eta\right] & \approx-1.46 \\
\cos \left[q_{29}\left(x_{25,2}^{+}\right) \eta\right] & \approx 2.53 & \cos \left[q_{29}\left(x_{25,2}^{-}\right) \eta\right] & \approx-0.5174
\end{aligned}
$$

These calculations show that the corrections to the upper and lower boundaries are both negative for $29 T M 1$ band, and both positive for $25 T M 2$ band in complete agreement with numerical calculations.

As it was mentioned we choose the supermodes $29 T M 1$ and $25 T M 2$ for illustration of our general results mostly because their dispersion curves were observed experimentally in Ref. 8 . However, before comparing the experimental results with our theory we should recall that the experiment in Ref 8 was conducted in a resonance configuration, when $q_{l}$ was fixed by the conditions of the experiment, and real and imaginary parts of frequency were measured via positions and widths of the respective resonances. This experimental setup is different from what was assumed in our theoretical analysis. Therefore, the comparison makes sense only away from the band boundaries, where decay rates are relatively small and the inverse of 


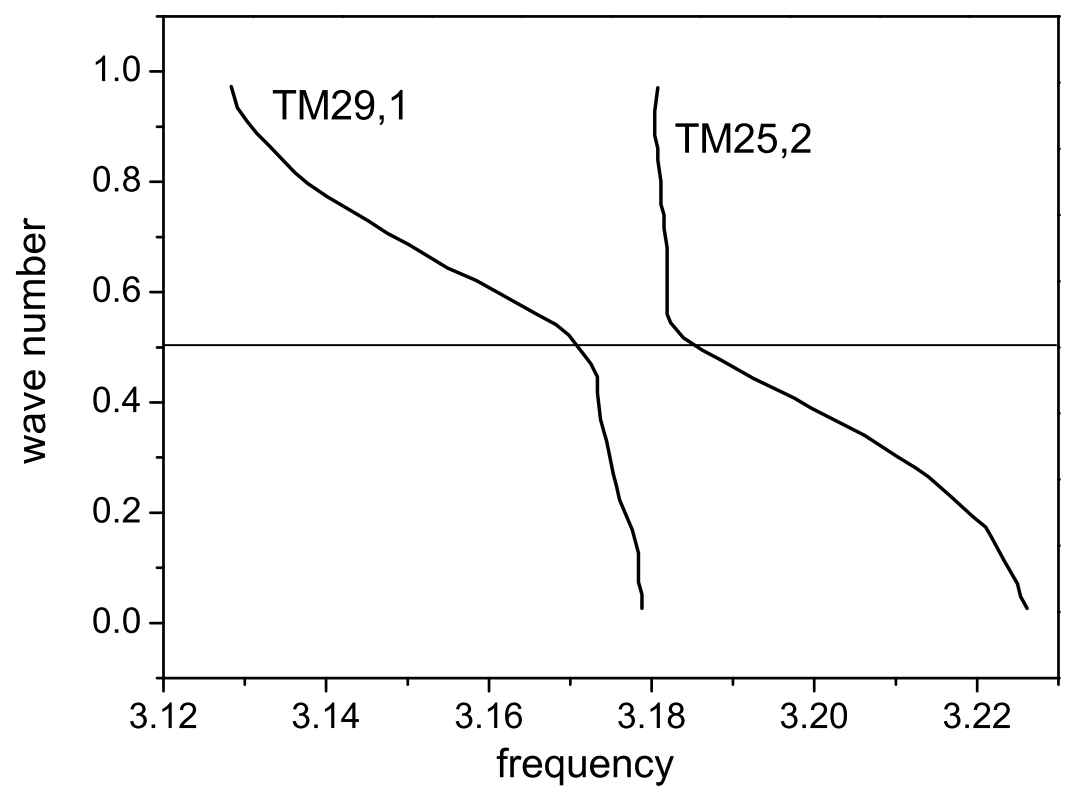

FIG. 5: Experimental dispersion curves of $29 T M 1$ and $T M 24,2$ bands obtained by digitizing one of the figures in $\operatorname{Ref} 8$.

the experimentally observed quantity $x_{l}(q)$ is close to $q_{l}(x)$ studied here. Fig 5 demonstrates the curves $q_{29,1}(x)$ and $q_{25,2}(x)$ produced by digitizing Fig.2b from Ref.8. Comparing this figure with results of our calculations, Fig 3 one can see an excellent qualitative agreement between the theory and the experiment.

\section{Strong inter-band coupling: band hybridization}

In order to illustrate the effects of the strong inter-band coupling we consider the interaction between modes $39 T E 1$ and $34 T E 2$. The resonance condition (32) for these modes is fulfilled at frequency $x_{r}$, which is within allowed spectral region for both bands, albeit rather close to their respective band boundaries. Therefore, we should expect the manifestation of the strong coupling effects in this case. Another important feature distinguishing this pair of modes is the different parity of their angular momentum indexes. As a result, the initial (single-band) dispersion curves of these supermodes are characterized in the vicinity of the resonance point by slopes of opposite signs. In the case of regular interacting modes 
such as phonon- or exciton-polaritons, one would expect in a situation like this a normal anti-crossing behavior resulting in the opening of the polariton band-gap in the spectrum of the system. This would have happened in the case considered here as well, if the interaction term in Eq.(31) were negative. It, however, is always positive as it was explained above and, therefore, no spectral gap arises. The shape of the dispersion curves emerging in this situation and shown in Fig [6 is rather unusual. The parts of the initial dispersion curves located below the crossing point are pushed downward from it and connect in a continuous manner to form a new hybrid band. The remaining parts of the initial dispersion laws are pushed upward such that the wave numbers corresponding to the resultant second dispersion curve become complex valued in the entire spectral region under consideration, with its real part remaining equal to $\pi$ for all considered frequencies. In other words, the spectral region covering initial $39 T E 1$ and $34 T E 2$ bands is a forbidden band for the second dispersion curve. Thus, the inter-band coupling turns two co-existing initial bands into a single hybrid band with rather unusual dispersion properties. Instead of a spectral gap we have here a region of forbidden values of wave numbers, which extends from the maximum value of the wave number on the lower dispersion curve, $q_{\max }$, all the way to the boundary of the Brillouin zone. This means that the states characterized by the wave numbers from the forbidden region cannot have a real valued frequency, i.e. do not correspond to stationary solutions of the respective dynamic equations.

The new allowed hybrid band is a combination of the initial bands for the two interacting supermodes and covers a spectral region previously occupied by $39 T E 1$ and $34 T E 2$. The shifts of the initial band boundaries (the low frequency boundary for $34 T E 2$ and higher frequency boundary for 39TE1) can be estimated with the help of the same expression (33) that we obtained for the weak coupling case. This is possible because these band boundaries lie far away from the resonance point and we can use perturbation theory. Taking into account that the sign of parameters $\kappa$ is now different for the two supermodes we find that the the low frequency boundary of $34 T E 2$ shifts toward higher frequencies by approximately 0.025, while the higher frequency boundary of 39TE1 supermode shifts toward lower frequencies by approximately 0.01 . These numbers are in good agreement with numerical calculations shown in Fig 6. This figure also shows that taking into account the reservoir modes (second iteration of our procedure) does not change properties of the hybrid mode too much.

While the existence of the wave number gap is the general property of the dispersion 


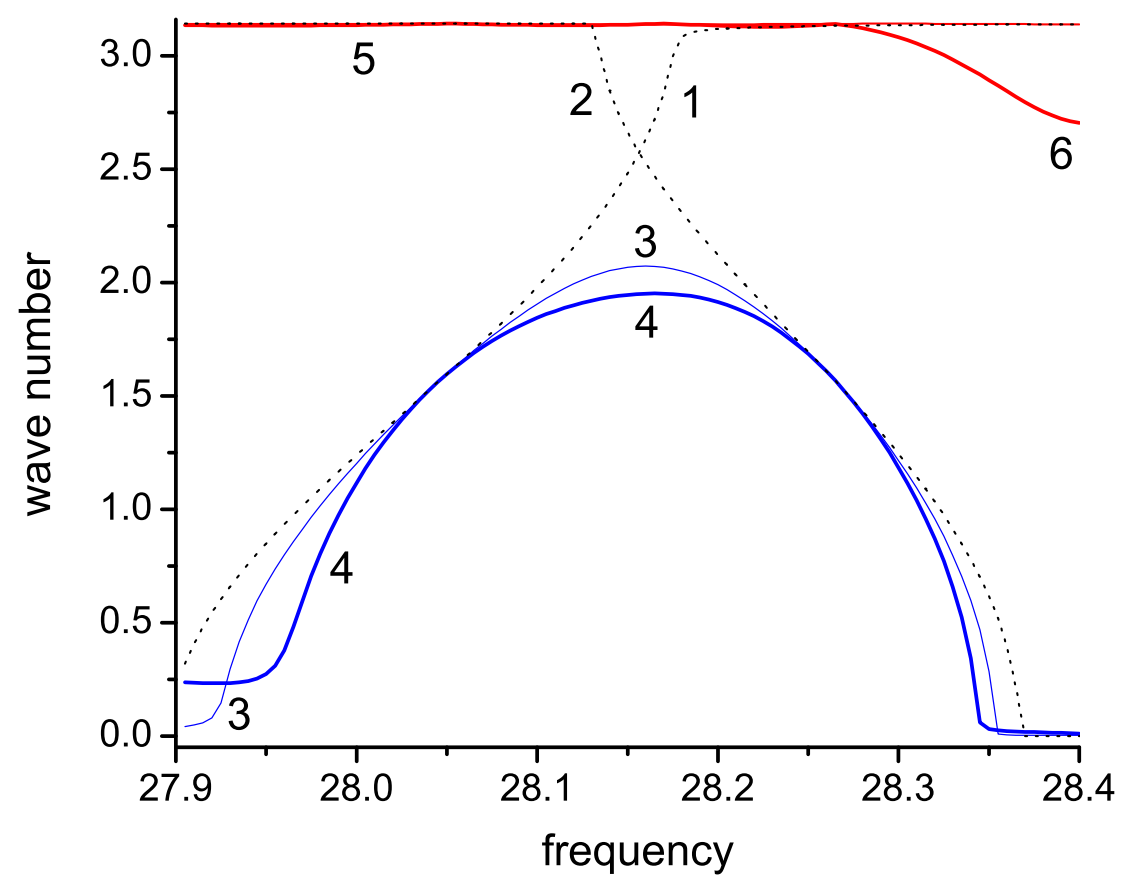

FIG. 6: Dispersion laws describing hybridized bands arising as a result of interaction between $39 T E 1$ and $34 T E 2$ supermodes. Curves 1 and 2 represents initial unperturbed dispersion laws, 3 and 5 are dispersion curves obtained without accounting for interaction with reservoir modes, and curves 4 and 6 depict dispersion laws of two branches with reservoir modes taken into account. The upper branch is almost completely pushed to the forbidden region.

equation (31) for the significant supermodes characterized by angular momentum index of different parity, the complete vanishing of the upper hybrid band is specific for the pairs of modes under consideration here. In order to understand the properties of these bands better we can estimate the distance between the lower and upper branches exactly at the resonance point neglecting the effects due to the reservoir modes. This distance, $\Sigma$, determines the position of the maximum $q_{\max }$ for the lower branch, and the minimum $q_{\min }$ for the upper branch. It is more convenient to work with $\cos q_{ \pm}$than with the wave numbers themselves, so we will define $\Sigma$ via equation:

$$
\begin{aligned}
& \cos \left(q_{\max } \eta\right)=\cos \left(q_{r} \eta\right)+\Sigma \\
& \cos \left(q_{\min } \eta\right)=\cos \left(q_{r} \eta\right)-\Sigma
\end{aligned}
$$


The magnitude of $\Sigma$ can be estimated from Eq.(31), which gives

$$
\Sigma=\frac{\operatorname{Re}\left[\sqrt{4 \Omega_{l_{1} l_{0}}-\Gamma^{2}-4 i \Omega_{l_{1} l_{0}} \Gamma}\right]}{1-\Omega_{l_{1} l_{0}}}
$$

where we took into account that the resonance occurs close to the band boundaries, so that $\operatorname{Re}\left[\cos q_{l_{0}}\right] \approx \operatorname{Re}\left[\cos q_{l_{1}}\right] \approx-1$, and introduced $\Gamma=\operatorname{Im}\left[\cos q_{l_{1}}\right]$, which represents the supermode with the largest radiative decay (34TE2 in the case under consideration). However, the radiative decay of the $34 T E 2$ supermode is still so small that $\Gamma^{2} \ll \Omega_{l_{0} l_{1}}$ in this case, and $\Sigma$ can be approximated as

$$
\Sigma=\frac{2 \sqrt{\Omega_{l_{1} l_{0}}}}{1-\Omega_{l_{1} l_{0}}}
$$

In the particular example of $39 T E 1$ and $34 T E 2$ bands the resonance occurs at a point, where $\cos q_{r}$ is so close to the boundary value of -1 , that $\cos \left(q_{\min } \eta\right)$ becomes less than -1 making the respective wave numbers for the entire frequency region imaginary. Should the crossing point of the two interacting supermodes lie farther away from the band boundaries, the part of the upper hybrid band could also survive, but it would be restricted by the frequency region in the vicinity of the resonance frequency. The $\cos \left(q_{\max } \eta\right)$ on the other hand is pushed farther away from the boundary, so that the entire lower band remains allowed, and the respective maximum allowed value of $q$ can be estimated as $q_{\max } \eta \approx q_{r} \eta-\sqrt{2 \Sigma}$. Comparison of these estimates with numerical results shows that they give a relatively good approximation for the respective quantities in the case under consideration.

One of the consequences of having a gap for wave numbers, is that formally speaking, the group velocity of the excitation described by the hybrid dispersion law diverges at $q=q_{\max }$. This form of a dispersion curve, therefore, raises a question if it is consistent with casuality. This question was discussed in Ref.19, where a similar form of the dispersion curve was found in an one-dimensional resonant photonic crystal. It was shown in that work that the wave-number gap and accompanying it infinite group velocity do not contradict causality, if attenuation of the respective excitation is properly accounted for. Similar anomalous behavior of the group velocity (without the wave number gap, of course) has been known for a long time, for instance, in the case of exciton-polaritons. ${ }^{20} \mathrm{~A}$ more general statement that abnormal behavior of group velocity should be expected in the regions of resonant absorption in all dispersive dielectrics was proven in Ref.21.

In spite of formal analogy between the anomalous dispersion law presented in Fig [6 and the results of Ref 19 these two situations significantly differ from each other. The wave 
number gap found in Ref 19 is caused by the frequency dispersion of the respective dielectric medium, and coincides with regions of anomalous dispersion and resonant absorption. The origin of the gap in our case is completely different and can be traced to the combination of two factors: different signs of the slopes of the initial dispersion curves and positive value of the respective coupling constant. The latter is caused, as we already discussed, by a non-Hermitian nature of the matrix describing the inter-band coupling in the case under consideration. Accordingly, the decay rate of the photonic supermodes considered here does not show any resonant enhancement in the vicinity of the wave-number gap. We present the frequency dependence of this quantity, which remains surprisingly small in all frequency range corresponding to the allowed hybrid band, and demonstrates a weak nonmonotonic dependence on frequency with a flat minimum (Fig[7). We can explain this behavior qualitatively by reminding that at the centers of both initial bands the interaction between them vanishes, so the decay rate at these two points coincides with the decay rates of the original WGM resonances. The crossing of the curves 2,3 and 4 in the insert in Fig:7 corresponds to the initial 34TE2 WGM, and the second (counting from the left) minimum on curve 4 corresponds to $x_{39 T E 1}$. The first minimum on this curve appears only after the interaction with reservoir modes is taken into account and presents another peculiarity of the system under consideration. Normally, one would expect that the reservoir bands can only increase the radiative rate, while here we observe an opposite behavior. A possible qualitative explanation of this effect can be offered on the basis of the following arguments. Let us assume, for an instance, that instead of considering the experimental situation, in which frequency is considered real and the wave number complex, we deal with the resonance type of experiment with real $q$ and complex $x$. In this case it is reasonable to assume, and the results of Ref 12 support this assumption, that the the reservoir modes would play its regular role and increase the width of the respective resonances, $\Delta x$. The transition from this description to the one used in this paper, with complex valued $q$ can then be approximately carried out with the help of the following expression: $\operatorname{Im} q \simeq(d \operatorname{Re} q / d x) \Delta x$. Thus, the frequency regions with flat dispersion curve should be characterized by decreasing imaginary part of the wave number. In order to understand why this minimum appears only after reservoir bands are taken into account, one needs to compare respective dispersion curves. From Fig [6] one can see that without the reservoir modes the maximum of the dispersion curve is relatively narrow. The reservoir modes make the maximum wider, which means 


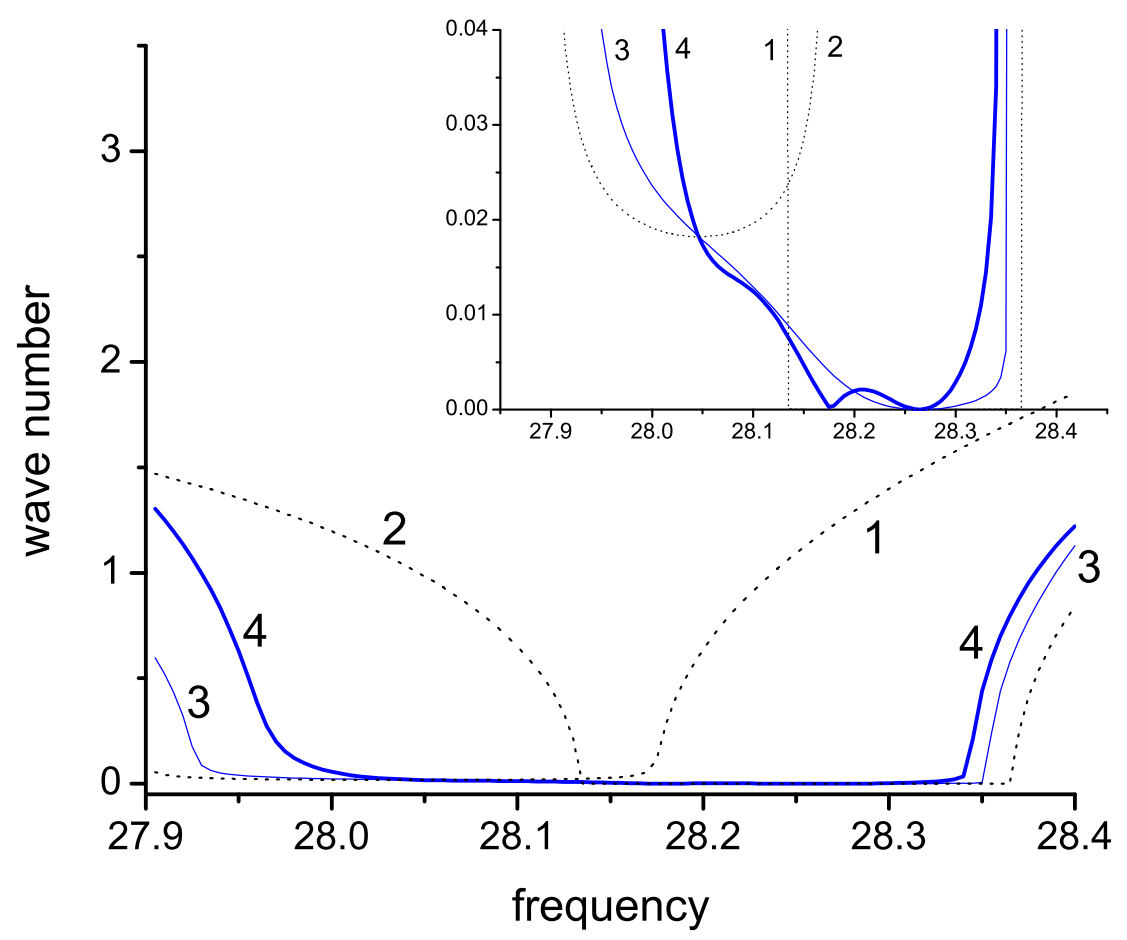

FIG. 7: Radiative decay rates for the allowed hybridized band. The labeling of the lines is the same as in Fig 6. The insert magnifies the region around the center of $39 T E 1$ band.

that a broader frequency interval is characterized by small values of $d \operatorname{Re} q / d x$. This causes a faster decrease of the spatial decay rate when frequency shifts toward the allowed region for former $39 T E 1$ band, which can be seen in Fig [7 This tendency reverses for frequencies greater than $x_{r}$, when the group velocity starts decreasing. When, however, the frequency approaches $x_{39 T E 1}$, the decay rate decreases again resulting in a curve with two minima, one at $x_{r}$, and the other one at $x_{39 T E 1}$.

\section{CONCLUSION}

In this paper we have developed a theory of the inter-band coupling in the system of dielectric spheres forming a one-dimensional chain. Our objective was to obtain analytical expressions allowing one to study band structure and dispersion properties of the collective excitations of this system with inter-band mixing effects taken into account. We developed an approximation scheme generalizing an approach of Ref.12, which was originally applied to 
the case of two coupled spheres and derived a general dispersion equation describing the band structure emerging as a result of the inter-band interaction. We showed that there might exist three qualitatively distinct regimes of such coupling depending upon the properties of the initial single-band dispersion curves of the interacting supermodes, and the strength of the interaction. Our general results were applied to two particular examples illustrating two of the possible manifestations of the inter-mode coupling.

As an illustration of the regime of weak band modification we considered frequency region corresponding to initial supermodes TE29, 1 and 25TM2, whose initial dispersion curves do not cross each other. Nevertheless, we showed that the inter-band coupling significantly modifies the dispersion curves of these supermodes and is responsible for their characteristic shape observed in experiments of Ref.8. What is interesting in this example is that the modification of the dispersion laws mimics the anti-crossing behavior typical for resonantly interacting excitations. At the same time, as it was mentioned above no crossing resonance takes place in this case. We explained the shape of these curves as a result of two effects. First, we showed that the position of frequency corresponding to the center of the band, where wave number $q=\pi /(2 \eta)$, where $\eta$ is dimensionless period of the structure, is pinned to the frequency of the parent WGM mode corresponding to a given band. Second, we demonstrated that the inter-band coupling shifts the boundary of the respective bands in the opposite directions. As a result, one of the dispersion curves bends upward, while the other one bends downward imitating the anti-crossing behavior. The results of the numerical calculations of these dispersion curves show very good qualitative agreement with experimental results of Ref 8 .

More interesting situation was found, however, in the spectral region corresponding to supermodes $39 T E 1$ and $34 T E 2$. These modes exhibit true crossing resonance very close to the boundaries of the both bands. It is also important that in the vicinity of the crossing point their initial dispersion curves have slopes of opposite signs. The interaction between these supermodes gives rise to a new hybrid band with a highly unusual dispersion characteristics. Instead of a standard avoiding crossing kind of behavior accompanied by opening of a gap in the frequency spectrum of the system, we found a gap in the allowed values of the wave numbers. More specifically, instead of two initial crossing dispersion curves, there emerges one curve characterized by a non-monotonic dependence of the wave number of frequency. The maximum value of the wave number is significantly lower than $\pi / \eta$, which 
determines traditional band boundaries. Thus, there is a range of wave numbers that do not correspond to excitations with any real valued frequency. Such a band structure is not related to the presence of the radiative decay, which remains very small throughout the entire band of frequencies corresponding to this hybrid band. We traced the origin of this effect to the non-Hermitian nature of the inter-band coupling typical for electromagnetic problems. The phenomenon of the wave number band-gap could be observed experimentally in an experiment similar to one carried out in Ref.8. In that experiment the fluorescent spectra were observed for chains with varying number of spheres. In normal situation, adding a sphere results in appearance of an additional peak on the spectrum so that each peak can be identified with a respective wave number. The wave number gap would manifest itself in this experiment as a failure of a new peak to appear after addition of a sphere to the chain. The dependence of the fluorescence spectrum upon the number of spheres in this situation is a non-trivial problem requiring a separate consideration. Other possible experimental manifestations of the predicted band structure may include unusual behavior of a pulse propagating along the chain, and angular dependence of the radiation emitted by the chain. More detailed analysis of these effects is also outside of the scope of this paper and will be carried out in the future.

\section{Acknowledgments}

We would like to express our appreciation of many fruitful discussions of this paper with A. Lisyansky, V. Menon, and E. Narimanov. One of us (A. R.) would like to thank Vladimir Shuvaev for help with numerical calculations.

1 A. Yariv, Y. Xu, R. K. Lee, and A. Scherer, Optics Letters 24, 711 (1999).

2 S. Mookherjea and A. Yariv, J. Sel. Top. Quantum Electron. 8, 448 (2002).

3 R. Chang and A. Campillo, eds., Optical Processes in Microcavities (World Scientific, Singapore, 1996).

4 S. Arnold, J. Comunale, W. B. Whitten, J. M. Ramsey, and K. A. Fuller, J. Opt. Soc. Am. B 9, 819 (1992). 
5 T. Mukaiyama, K. Takeda, H. Miyazaki, Y. Jimba, and M. Kuwata-Gonokami, Phys. Rev. Lett. 82, 4623 (1999).

6 Y. P. Rakovich, J. F. Donegan, M. Gerlach, A. L. Bradley, T. M. Connolly, J. J. Boland, N. Gaponik, and A. Rogach, Phys. Rev. A 70, 051801 (2004).

7 B. M. Moeller, U. Woggon, and M. Artemeyev, Opt. Lett. 30, 2116 (2005).

8 Y. Hara, T. Mukaiyama, K. Takeda, and M. Kuwata-Gonokami, Phys. Rev. Lett. 94, 203905 (2005).

9 V. Astratov, J. Franchak, and S. Ashili, Appl. Phys. Lett. 85, 5508 (2004).

10 K. A. Fuller, Applied Optics 30, 4716 (1991).

11 E. Lidorikis, M. M. Sigalas, E. N. Economou, and C. M. Soukoulis, Phys. Rev. Lett. 81, 1405 (1998).

12 H. Miyazaki and Y. Jimba, Phys. Rev. B 62, 7976 (2000).

13 J. A. Stratton, Electromagnetic theory (McGraw-Hill Book Company, New York and London, 1941).

14 S. Stein, Q. Appl. Math. 19, 15 (1961).

15 O. Cruzan, Q. Appl. Math. 20, 33 (1962).

16 W. Harrison, Elementary Electronic Structure (Wolrd Scientific, Singapore, 1999).

17 P. Vogl, H. P. Hjalmarsons, and J. D. Dow, J. Phys. Chem. Solids 44, 365 (1983).

18 A. Moroz, Phys. Rev. B 51, 2068 (1995).

19 O. Toader and S. John, Phys. Rev. E 70, 046605 (2004).

20 A. Puri and J. L. Birman, Phys. Rev. Lett. 47, 173 (1981).

21 E. L. Bolda, R. Y. Chiao, and J. C. Garrison, Phys. Rev. A 48, 3890 (1993). 